\title{
BMJ Open Systematic review with a meta-analysis: clinical effects of statins on the reduction of portal hypertension and variceal haemorrhage in cirrhotic patients
}

\author{
Sizhe Wan, Chenkai Huang, Xuan Zhu
}

To cite: Wan S, Huang C, Zhu X. Systematic review with a meta-analysis: clinical effects of statins on the reduction of portal hypertension and variceal haemorrhage in cirrhotic patients. BMJ Open 2019;9:e030038. doi:10.1136/ bmjopen-2019-030038

- Prepublication history and additional material for this paper are available online. To view please visit the journal (http:// dx.doi.org/10.1136/bmjopen2019-030038).

Wan $\mathrm{S}$ and Huang $\mathrm{C}$ contributed equally.

Received 27 February 2019

Revised 23 May 2019

Accepted 30 May 2019

Check for updates

(C) Author(s) (or their employer(s)) 2019. Re-use permitted under CC BY-NC. No commercial re-use. See rights and permissions. Published by BMJ.

Department of Gastroenterology, The First Affiliated Hospital of Nanchang University, Nanchang, China

Correspondence to Professor Xuan Zhu; jyyfyzx@163.com

\section{ABSTRACT}

Background Statins may improve outcomes in patients with cirrhosis. We performed a systematic review and meta-analysis to evaluate the effect of statins on patients with cirrhosis and related complications, especially portal hypertension and variceal haemorrhage.

Methods Studies were searched in the PubMed, Embase and Cochrane library databases up to February 2019. The outcomes of interest were associations between statin use and improvement in portal hypertension (reduction $>20 \%$ of baseline or $<12 \mathrm{~mm} \mathrm{Hg}$ ) and the risk of variceal haemorrhage. The relative risk (RR) with a $95 \% \mathrm{Cl}$ was pooled and calculated using a random effects model. Subgroup analyses were performed based on the characteristics of the studies.

Results Eight studies (seven randomised controlled trials (RCTs) and one observational study) with 3195 patients were included. The pooled RR for reduction in portal hypertension was $1.91\left(95 \% \mathrm{Cl}, 1.04\right.$ to $\left.3.52 ; \mathrm{I}^{2}=63 \%\right)$ in six RCTs. On subgroup analysis of studies that used statin for 1 month, the RR was $2.01(95 \% \mathrm{Cl}, 1.31$ to $\left.3.10 ; I^{2}=0 \%\right)$; the pooled RR for studies that used statins for 3 months was $3.76\left(95 \% \mathrm{Cl}, 0.36\right.$ to $\left.39.77 ; I^{2}=75 \%\right)$; the pooled RR for studies that used non-selective betablockers in the control group was $1.42(95 \% \mathrm{Cl}, 0.82$ to $2.45 ; I^{2}=64 \%$ ); the pooled RR for studies that used a drug that was not reported in the control group was 4.21 (95\% Cl, 1.52 to $11.70 ; \mathrm{I}^{2}=0 \%$ ); the pooled RR for studies that used simvastatin was $2.20(95 \% \mathrm{Cl}, 0.92$ to 5.29 ; $\left.\mathrm{I}^{2}=69 \%\right)$; RR for study using atorvastatin was $1.82(95 \%$ $\mathrm{Cl}, 1.00$ to 3.30). For the risk of a variceal haemorrhage, the RR based on an observational study was $0.47(95 \% \mathrm{Cl}$, 0.23 to 0.94$)$; in two RCTs, the pooled RR was 0.88 (95\% $\mathrm{Cl}, 0.52$ to $1.50 ; \mathrm{I}^{2}=0 \%$ ). Overall, the summed RR was 0.64 (95\% Cl, 0.42 to $\left.0.99 ; l^{2}=6 \%\right)$.

Conclusion Statins may improve hypertension and decrease the risk of variceal haemorrhage according to our assessment. However, further and larger RCTs are needed to confirm this conclusion.

\section{INTRODUCTION}

Cirrhosis is increasingly prevalent worldwide, as a result of a variety of chronic liver diseases.
Strengths and limitations of this study

- This will be the most comprehensive review of published and unpublished data of clinical effects of statins on the reduction of portal hypertension and variceal haemorrhage.

- This systematic review provides strong evidence for clinicians using statins to treat portal hypertension and variceal haemorrhage.

- Eligible studies screening, data extraction and quality assessment were performed by two independent reviewers to reduce the potential for reviewer bias.

- Large randomised controlled trials are needed to confirm beneficial effects of statins in patients with liver diseases.

- Only studies in the English language have been included in the analysis.

Cirrhosis, including compensate and decompensate, was in the top eight causes of death in the USA in 2010 and led to more than 49500 deaths. ${ }^{1}$ The median survival of patients with compensated cirrhosis is $>12$ years, and patients with decompensated cirrhosis exhibit a median survival of $<2$ years. ${ }^{2}$ Portal hypertension and oesophageal varices are common complications of cirrhosis, and these conditions develop into variceal haemorrhage, which produces a mortality of $10 \%-15 \%$ per episode. ${ }^{3}$ The hepatic venous pressure gradient (HVPG) is a significant indicator of portal hypertension and varices bleeding. Reduction in HVPG indicates an improvement in portal hypertension and a decline in bleeding risk. ${ }^{4}$

Statins are widely used in clinical practice because of their exact and effective lipid-lowering effects. ${ }^{56}$ The use of statins in patients with liver disease has long been limited by concerns of their potential hepatotoxicity, which have been raised by anecdotal 


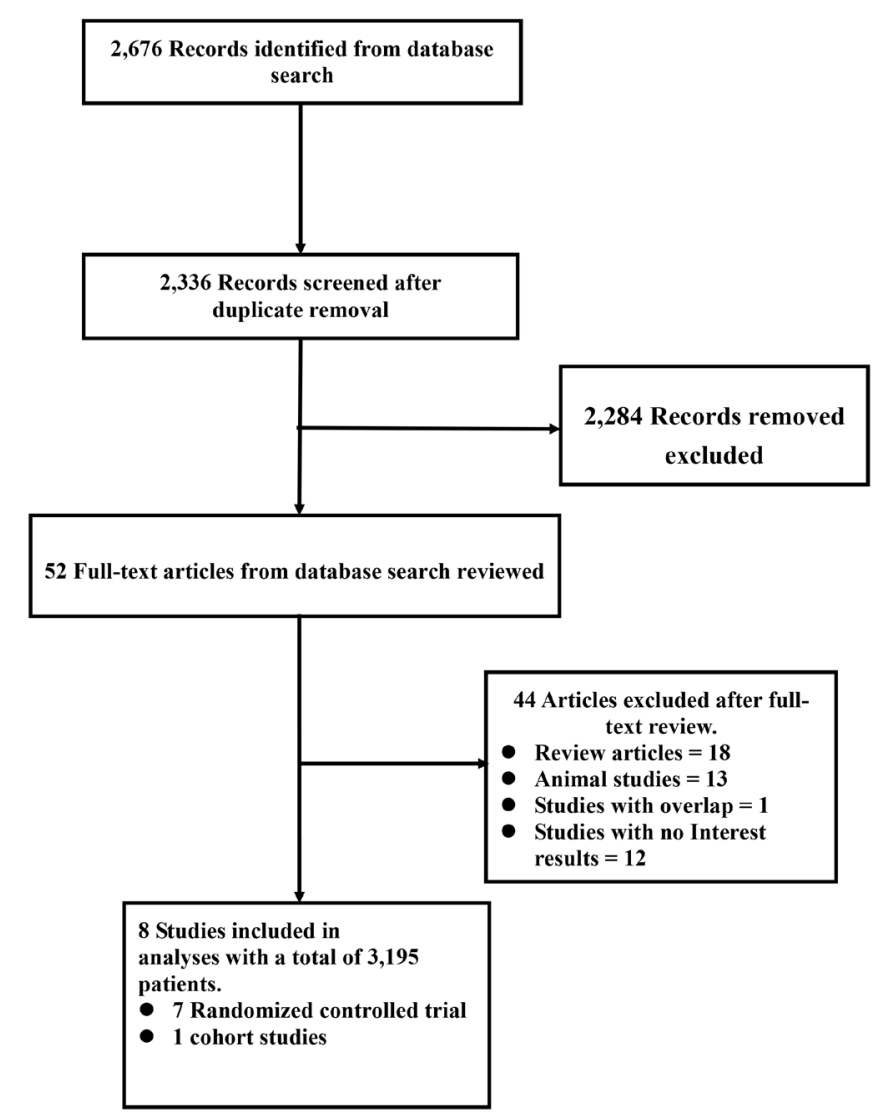

Figure 1 Preferred Reporting Items for Systematic Reviews and Meta-Analyses flowchart.

evidence of increased liver enzymes following statin use or the possible trapping of lipids in the liver. ${ }^{7}$ Some residual concern remains among primary care physicians in prescribing statins to patients with underlying liver disease because some of doctors still believe that these patients are at increased risk for hepatotoxicity. ${ }^{8}$ However, a growing interest in the potential benefits of statins in patients with liver diseases has recently emerged. ${ }^{7-12}$ Recent in vivo and in vitro experiments have gradually demonstrated that statins also exhibit anti-inflammatory, ${ }^{13}$ immune-modulating, ${ }^{14}$ antiproliferative ${ }^{15}$ and antioxidant ${ }^{16}$ effects as well as improved endothelial function ${ }^{17}$ and inhibit platelet aggregation ${ }^{18}$ and certain Gram-negative bacteria. ${ }^{19} 20$ These findings led to the development of statins in basic research of liver disease and laid a solid foundation for clinical practice.

We performed a systematic review and meta-analysis based on the most recent studies (randomised controlled trials (RCTs) and a cohort study) to evaluate the effects of statins in patients with cirrhosis and related complications, especially portal hypertension and variceal haemorrhage.

\section{METHODS}

\section{Search strategy}

PubMed, Embase, Cochrane Controlled Trial Registry and The Cochrane Library were searched up to February
2019 to identify all relevant articles on the effect of statins in liver cirrhosis and retrieve pertinent studies (online supplementary method). No language restrictions were imposed. An experienced medical librarian designed and implemented the search strategy. Electronic databases were searched using the following search terms: liver cirrhosis, ascites, portal hypertension, statin, hydroxymethylglutaryl-CoA reductase inhibitors. The detailed search strategy is available in the 'online supplementary method'. Two reviewers (SW and $\mathrm{CH}$ ) independently assessed the titles and abstracts of the studies that met the eligibility criteria for inclusion.

\section{Data abstraction}

Two reviewers (SW and $\mathrm{CH}$ ) independently extracted the data. The following data were collected from each study: year of publication, study design, inclusion criteria, exclusion criteria, aetiology of cirrhosis, total number of patients in each group, primary outcome reported and Child-Turcotte-Pugh (CTP) class and ascites. Any divergence between the reviewers was discussed with a third reviewer (XZ), and agreement was reached by consensus.

We used the Newcastle-Ottawa scale to determine the quality of the cohort studies, and the Cochrane tool was used to determine the risk of bias for RCTs.

\section{Outcomes assessed}

Our primary outcome of interest was the association between statin use and the reduction in portal hypertension. The secondary outcomes of interest were the association between statin use and variceal bleeding. Several subgroup analyses were performed based on the quality of the studies, medication time, types of drugs in the control group and types of statins. The adverse effects of statins were not included in the study due to insufficient information.

\section{Quality of evidence}

We used the Grading of Recommendations Assessment, Development, and Evaluation (GRADE) framework to evaluate the quality of the evidence. ${ }^{21}$ The GRADE approach for systematic reviews defines the quality of a body of evidence as the extent to which one can be confident that an estimate of an effect or association is close to the quantity of specific interest. The following factors were considered in determining the quality of evidence: risk of bias, directness of evidence, heterogeneity, precision of effect estimates and risk of publication bias.

\section{Statistical analysis}

The trials and patient characteristics are reported as the number of observations and proportions. The relative risk (RR) and 95\% CI that achieved a target haemodynamic response in each group were pooled using the DerSimonian and Laird random effects model. ${ }^{22}$ Intertrial heterogeneity was statistically assessed using the chi-square test and is expressed as the $\mathrm{I}^{2}$ value, and $\mathrm{I}^{2}$ values $>50 \%$ were reflective of substantial heterogeneity. ${ }^{23} \mathrm{~A}$ formal 


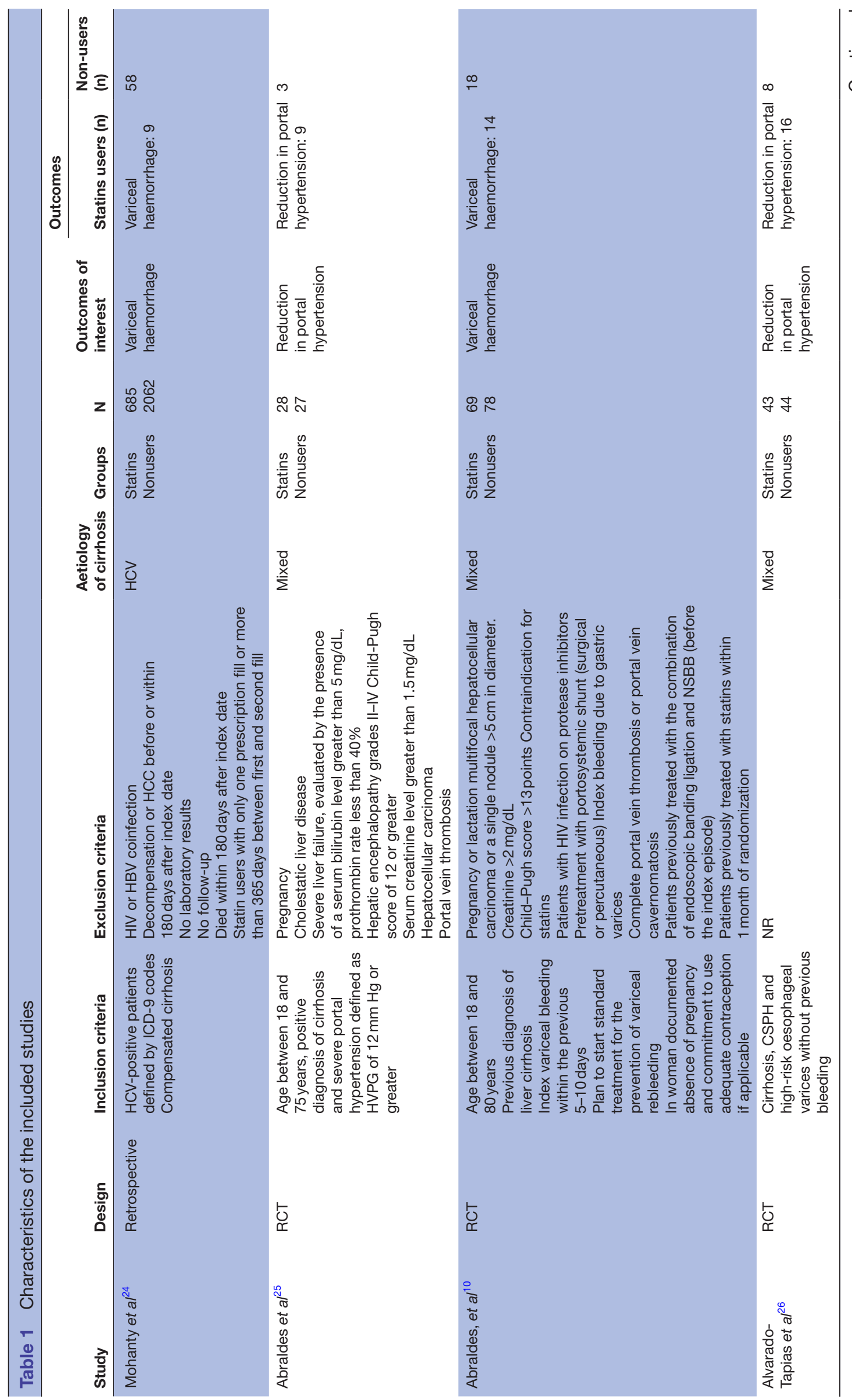

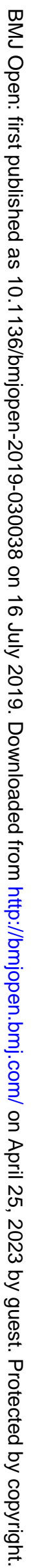




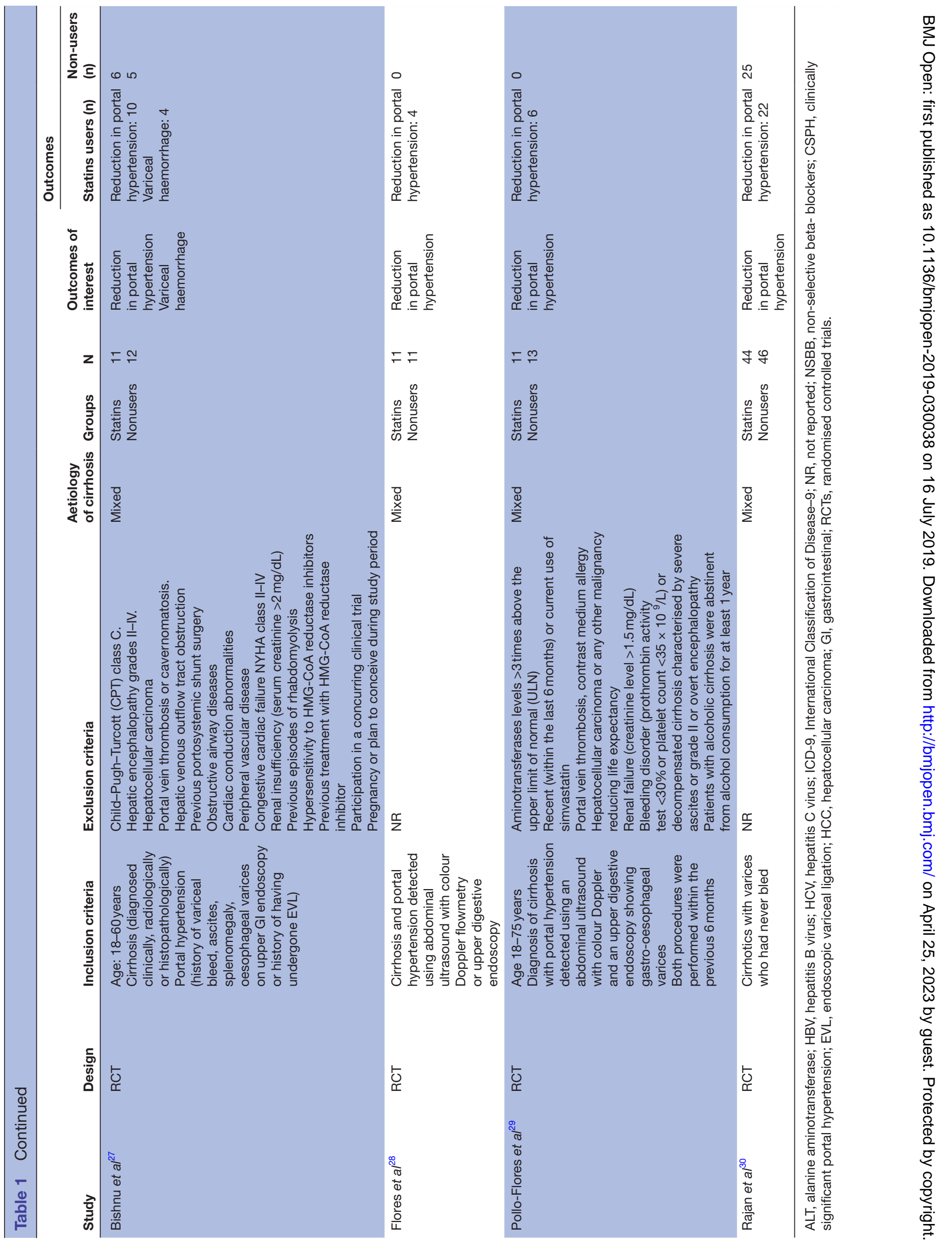


assessment of publication bias using the egger test was performed (online supplementary figure 1).

\section{Patient and public involvement}

This meta-analysis did not involve patients or the public.

\section{RESULTS}

\section{Search results}

A total of 2676 potentially eligible references were retrieved in the literature search, and 2624 were excluded based on the titles and abstracts. A further 44 articles, referred to as full articles, were deemed ineligible. Twelve studies were excluded because they did not clearly report the number of patients with improved portal hypertension and variceal haemorrhage. Eight studies with a total of 3195 patients met our inclusion criteria and were included in our meta-analysis (seven RCTs and one observational study). ${ }^{1024-30} \mathrm{Six}$ studies included patients who exhibited the target reduction in HVPG $>20 \%$ from baseline or $<12 \mathrm{~mm} \mathrm{Hg}$ in the statins group. Three studies included events of variceal bleeding in patients with cirrhosis. Figure 1 summarises the search strategy.

\section{Description of included studies}

Table 1 shows the characteristics of these studies. These studies included 3195 liver cirrhosis patients, of whom 902 patients were exposed to statins. One study was performed exclusively in patients with HCV mono-infection, and seven studies included cirrhosis with multiple underlying aetiologies. The medication time of statins was 1 month in three studies. However, statins were used for 3 months in three studies. Six studies provided the desired data as regarded decrease in HVPG (reduction $>20 \%$ or $<12 \mathrm{~mm} \mathrm{Hg}$ ).

The only observational study was of high quality, as exhibited by the high Newcastle-Ottawa quality score. Table 2 summarises the methodological qualities of the observational study and RCTs. Figure 2 shows the methodological qualities of the RCTs.

Table 3 summarises the characteristics of the 3195 patients included in the eight studies. Statin users and nonusers were generally male because the cirrhosis incidence in females was lower than that in males. Patients were mostly categorised as CTP A and B classes, 221 of $255(87 \%)$ in two studies. No appreciable differences in the complications of cirrhosis, such as ascites or previous variceal bleeding, were observed between the two study groups across the eight studies.

\section{Outcome evaluation}

Improvement in portal hypertension

Six studies including 301 patients evaluated the improvement in portal hypertension in cirrhosis. Overall, a decrease in HVPG ( $>20 \%$ from baseline or $<12 \mathrm{~mm} \mathrm{Hg}$ ) was achieved with statins in 57 of 135 evaluable patients compared with 36 of 141 patients in the control group (RR, $1.91 ; 95 \% \mathrm{CI}, 1.04$ to $\left.3.52 ; \mathrm{I}^{2}=63 \%\right)$. Three subgroup

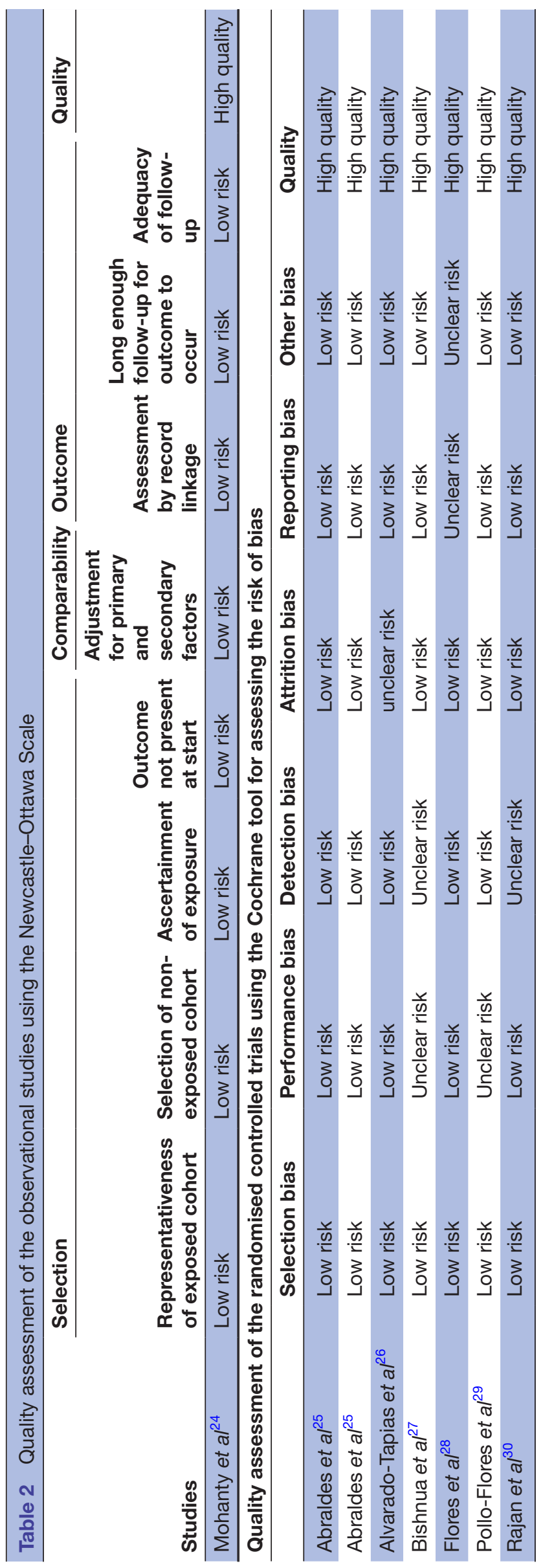




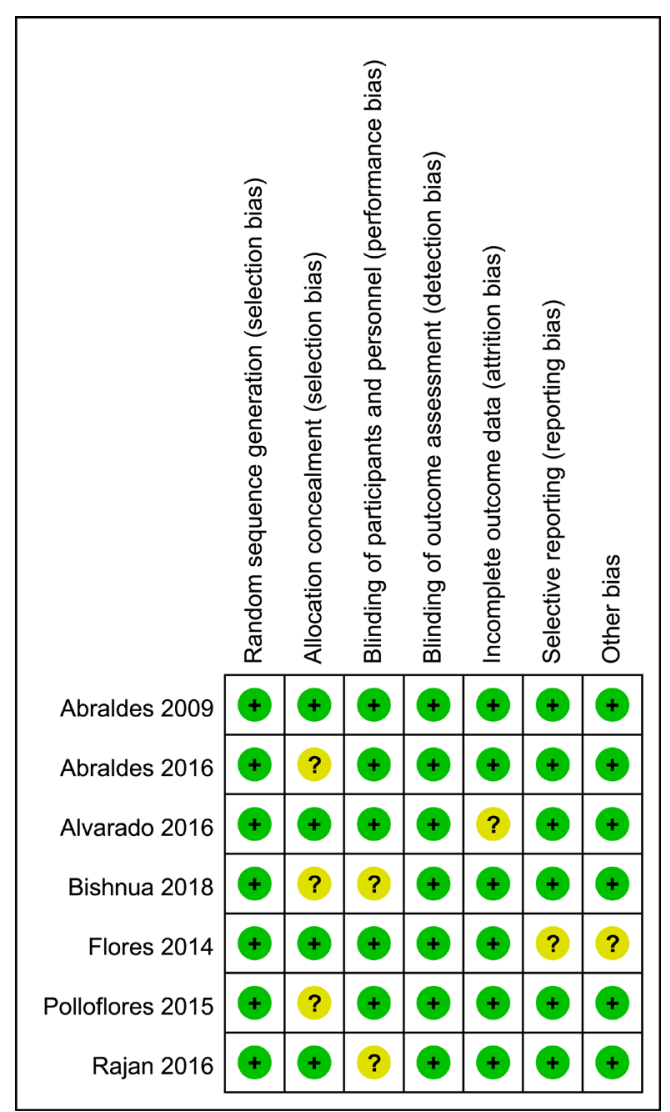

Figure 2 Risk of bias assessed using the Cochrane risk of bias tool for randomised controlled trials. analyses were performed, based on the medication time, types of drug used in the control group and types of statins. Subgroup analysis of the medication time of statins included three studies that used statins for 1 month (RR, $2.01 ; 95 \% \mathrm{CI}, 1.31$ to $\left.3.10 ; \mathrm{I}^{2}=0 \%\right)$ and three studies that used statins for 3 months (RR, 3.76; 95\% CI, 0.36 to 39.77; $\mathrm{I}^{2}=75 \%$ ) (figure 3 ). The second subgroup analysis was based on the types of drugs used in the control group, including non-selective beta-blockers (NSBB) and not explicitly reported drugs. The pooled RR for NSBB users was $1.42\left(95 \% \mathrm{CI}, 0.82\right.$ to $\left.2.45 ; \mathrm{I}^{2}=64 \%\right)$, and the pooled RR for the not explicitly reported drugs was $4.21(95 \%$ CI, 1.52 to $11.70 ; \mathrm{I}^{2}=0 \%$ ) (figure 4 ). The third subgroup analysis was based on the types of statins. Five studies used simvastatin (RR, 2.20; $95 \% \mathrm{CI}, 0.92$ to $5.29 ; \mathrm{I}^{2}=69 \%$ ), and one study used atorvastatin (RR, 1.82; 95\% CI, 1.00 to 3.30) (figure 5).

There was moderate persuasion supporting the use of statins associated with an improvement in portal hypertension based on the RCTs. However, the result was limited by the study size (109 events in 301 patients).

Risk of variceal haemorrhage

Three studies including 3025 patients evaluated the association between statin use and the occurrence of variceal bleeding. Overall, 27 events occurred in 765 statin users, and 81 events were reported in 2152 nonusers. A subgroup analysis was performed based on the type of trial. Overall, the pooled RR for the risk of variceal haemorrhage was

Table 3 Characteristics of participants in the included studies

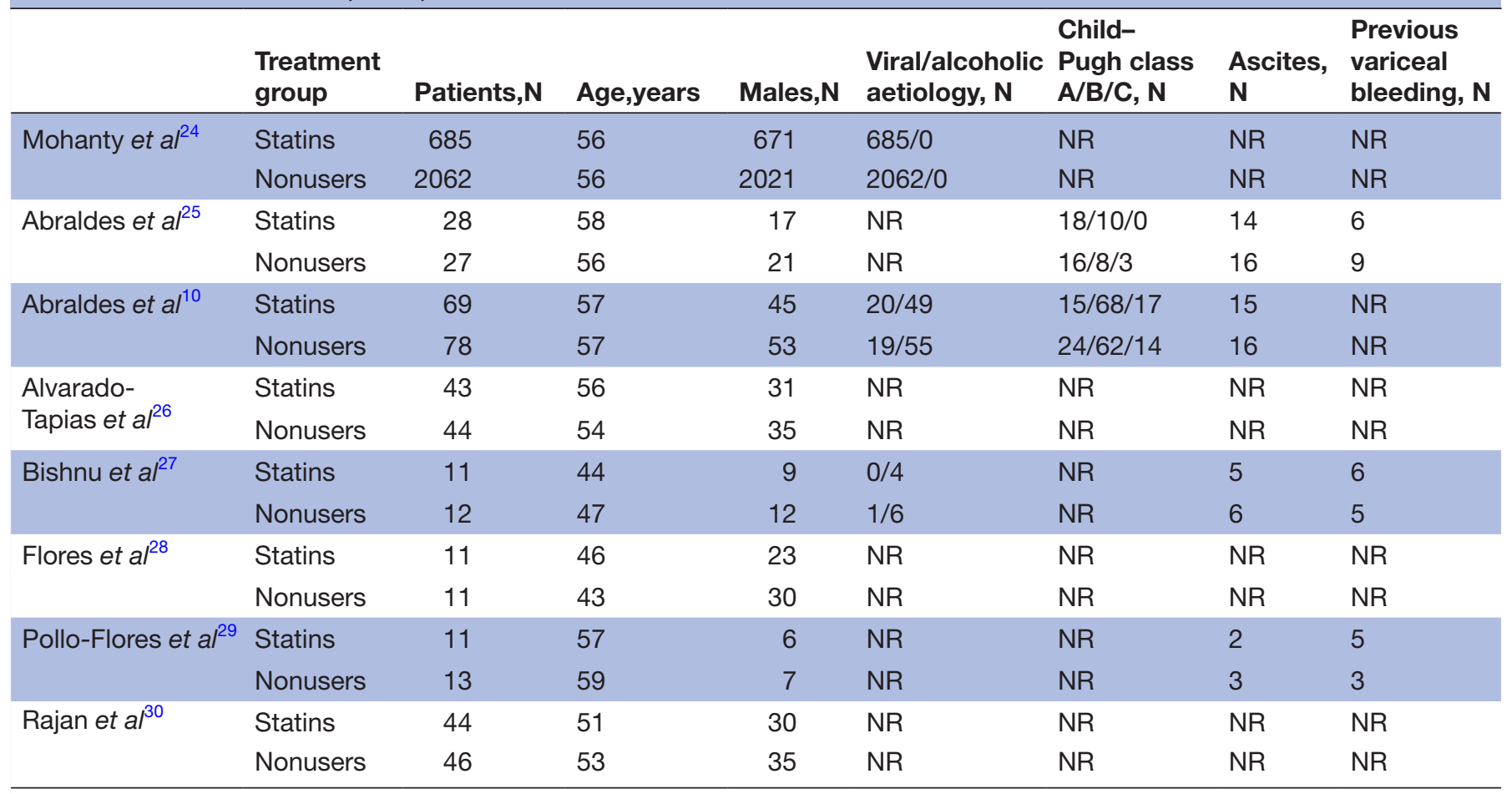

NR, not reported. 


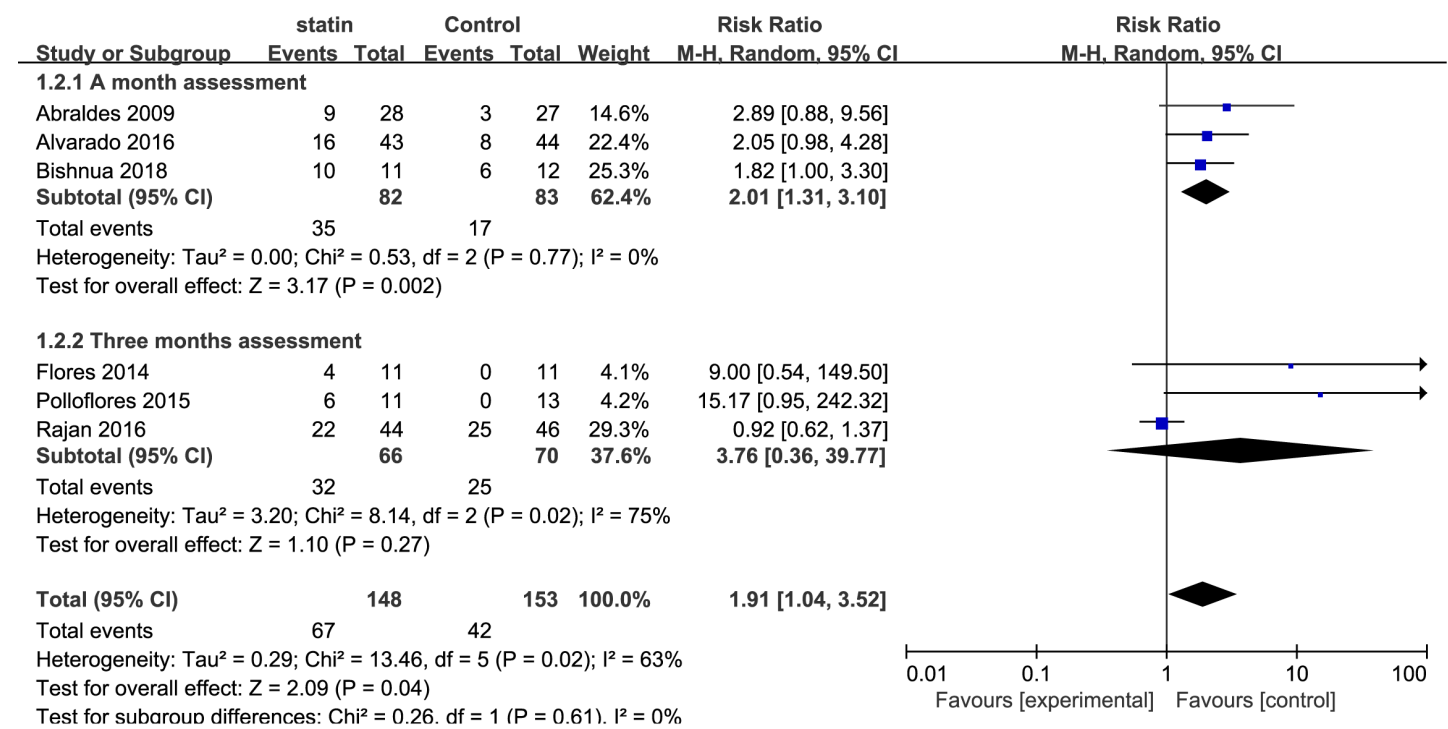

Figure 3 Forest plot using the Mantel-Haenszel $(\mathrm{M}-\mathrm{H})$ analysis method to evaluate the role of statins in the reduction of portal hypertension using a subgroup analysis based on medication time of statins.

$0.64\left(95 \%\right.$ CI, 0.42 to $\left.0.99 ; \mathrm{I}^{2}=6 \%\right)$. The RR for the only one observational study was 0.47 (95\% CI, 0.23 to 0.94 ). The pooled RR for the two RCTs studies was $0.88(95 \%$ CI, 0.52 to $1.50 ; \mathrm{I}^{2}=0 \%$ ) (figure 6 ).

\section{DISCUSSION}

This meta-analysis demonstrated the possible roles of statin use in patients with cirrhosis against the development of portal hypertension and the occurrence of variceal haemorrhage in eight studies (seven RCTs and one cohort study). The availability of statins was proven to lead to the decrease in portal hypertension and variceal bleeding across all trials. The summary RR between the numbers of HVPG reductions achieved in statin users and nonusers was 1.91 (95\% CI, 1.04 to $\left.3.52 ; \mathrm{I}^{2}=63 \%\right)$ in favour of statins. We performed three subgroup analyses because of the substantial heterogeneity. The subgroup analysis based on the medication time of statin use supported the improvement in portal pressure at the 1-month assessment (RR, 2.01; 95\% CI, 1.31 to $3.10 ; \mathrm{I}^{2}=0 \%$ ). However, this effect was not statistically significant at the 3-month assessment (RR, 3.76; 95\% CI, 0.36 to $39.77 ; \mathrm{I}^{2}=75 \%$ ). These results suggest that the effects of statins are not dose-dependent and lead to strong curative effects in patients who used statins for 1 month compared with patients with a longer duration of use. Several possible mechanisms may explain the biological plausibility of our findings. The hepatotoxicity of statins occurs via regulation of the P450 cytochrome in immune-mediated liver damage, which activates apoptosis and $\mathrm{T}$ cell-induced liver injury. ${ }^{31}{ }^{32}$ Previous clinical research ${ }^{33-36}$ confirmed these observations, which offset the benefits of statins over a longer treatment period. No considerable differences were observed in subgroup analyses for the use of

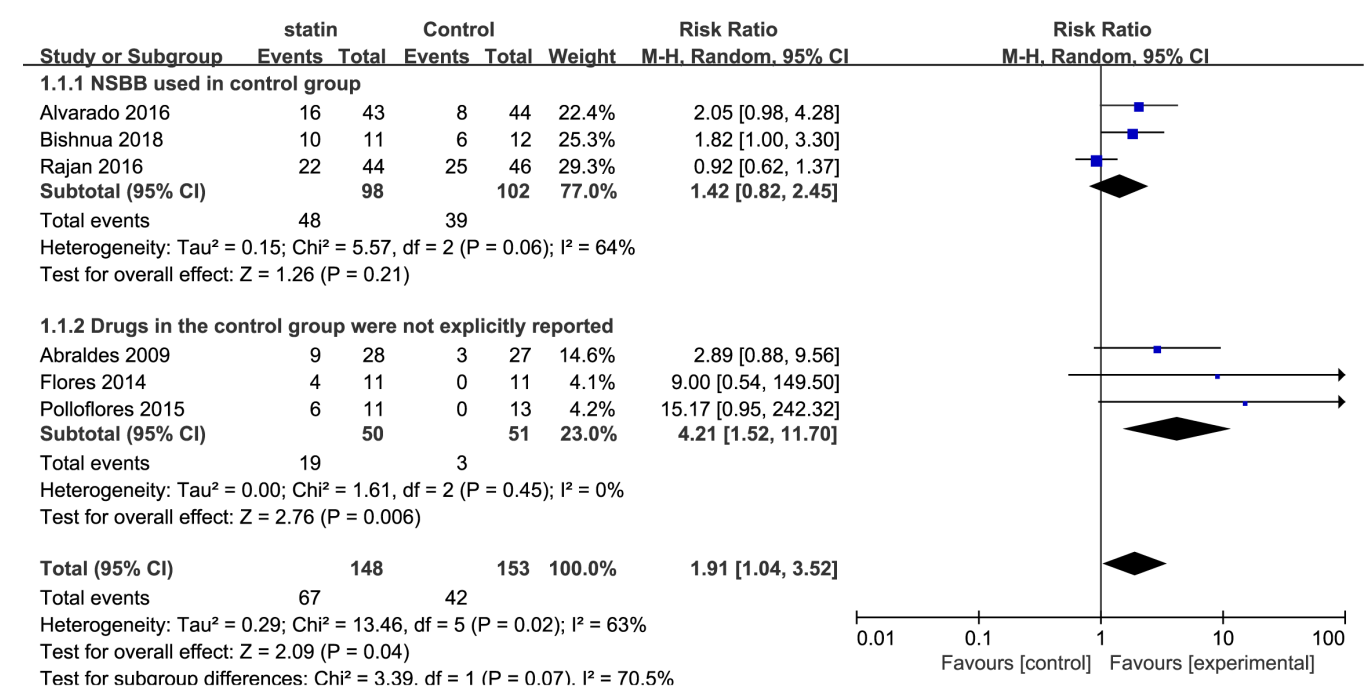

Figure 4 Forest plot using the Mantel-Haenszel $(\mathrm{M}-\mathrm{H})$ analysis method to evaluate the role of statins in the reduction of portal hypertension using subgroup analysis based on the types of drugs in the control group. NSBB, non-selective beta-blockers. 


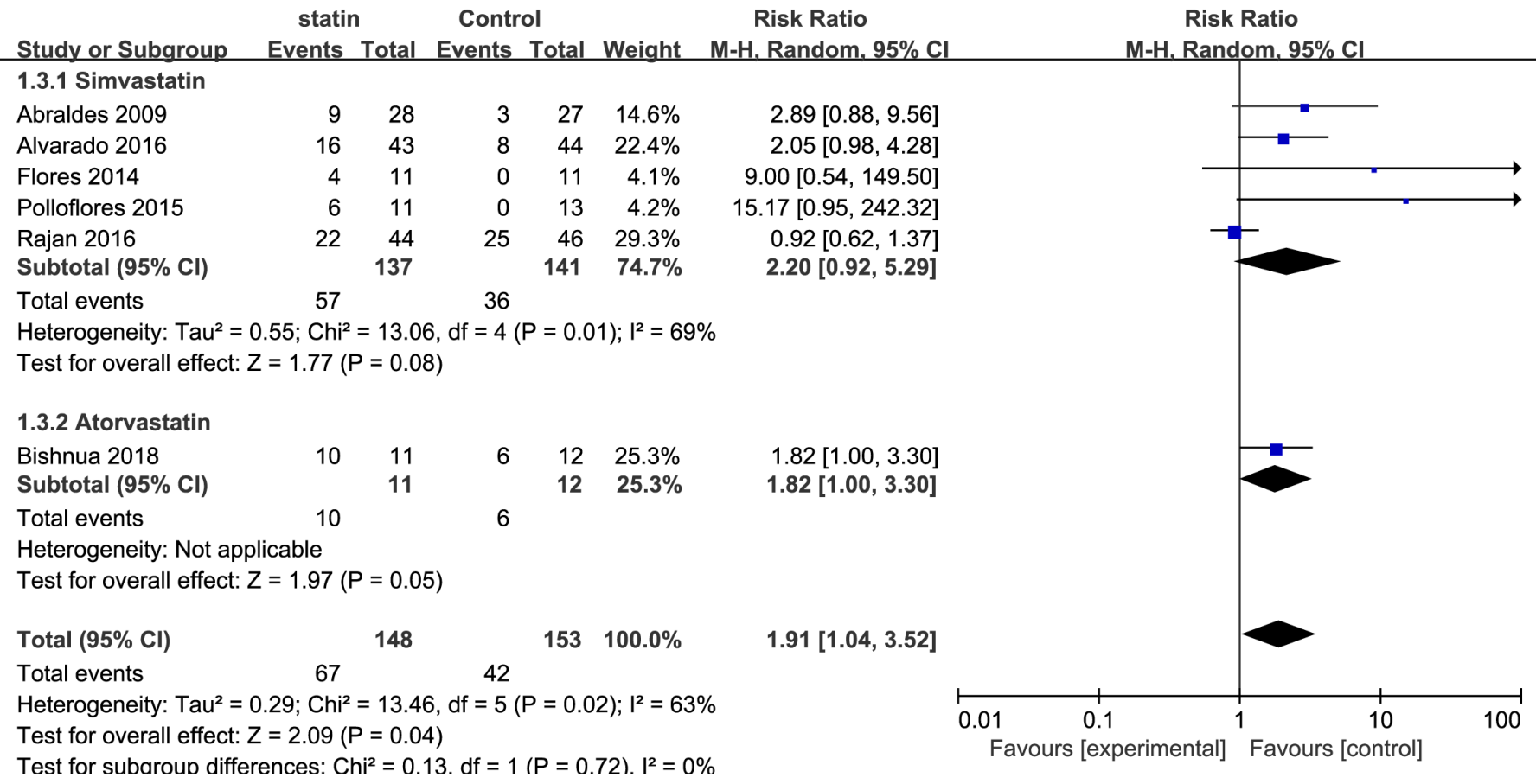

Figure 5 Forest plot using the Mantel-Haenszel $(\mathrm{M}-\mathrm{H})$ analysis method to evaluate the role of statins in the reduction of portal hypertension using subgroup analysis based on types of statins.

NSBB in the control group (RR, 1.42; 95\% CI, 0.82 to 2.45 ; $\left.\mathrm{I}^{2}=64 \%\right)$. We presume that improvements of portal hypertension by NSBB is the underlying mechanism. NSBB is clinically used to treat portal hypertension because of its efficacy in decreasing HVPG and variceal haemorrhage. ${ }^{37-40}$ Therefore, the use of NSBB in the control group may lead to no significant difference between the statin user and nonuser groups. Different types of statins exhibit inconsistent pharmacological actions. Therefore, patients were stratified by the statin varieties. The pooled
$\mathrm{RR}$ in a subset of patients who received simvastatin was 2.20 (95\% CI, 0.92 to $5.29 ; \mathrm{I}^{2}=69 \%$ ), which indicates no improvement. Atorvastatin users exhibited a decrease in portal pressure (RR, 1.82; 95\% CI, 1.00 to 3.30). Highquality evidence was included, but discrepancies, such as the medication time, may have led to imprecision.

Events of variceal haemorrhage were satisfactorily reported in three studies. The effect of statins on variceal bleeding as a common cause of death in patients with portal hypertension was also investigated. The pooled

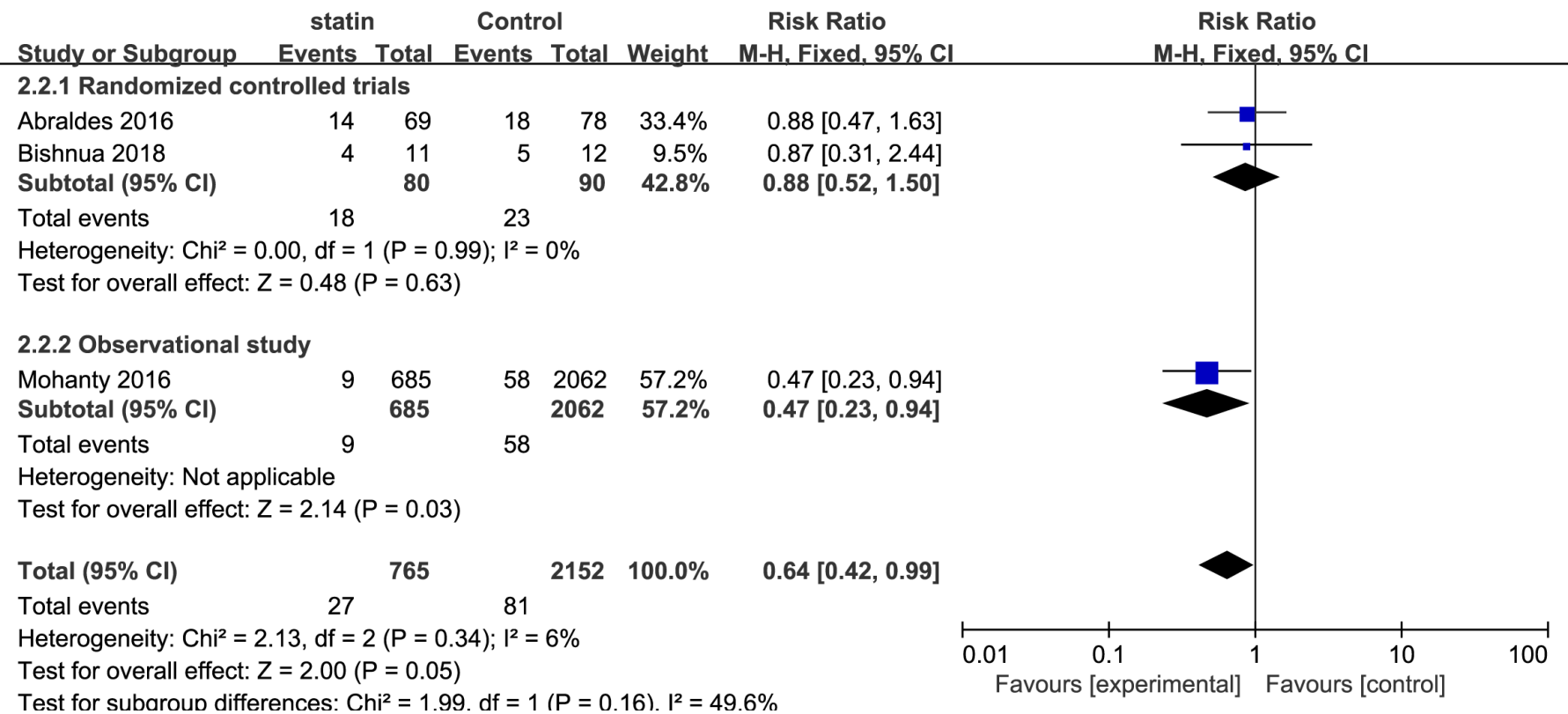

Figure 6 Forest plot using the Mantel-Haenszel $(\mathrm{M}-\mathrm{H})$ analysis method to evaluate the role of statins in the reduction of the risk of variceal haemorrhage using subgroup analysis based on types of statins. 
RR was 0.64 (95\% CI, 0.42 to $0.99 ; \mathrm{I}^{2}=6 \%$ ). However, the reduction in the pooled RR of the risk of variceal haemorrhage failed to reach statistical significance with statin use in two RCTs (RR, $0.88 ; 95 \%$ CI, 0.52 to $1.50 ; \mathrm{I}^{2}=0 \%$ ). Notably, the only observational study confirmed the superiority of statins in lowering the risk of variceal bleeding (RR, $0.47 ; 95 \% \mathrm{CI}, 0.23$ to 0.94 ). The characteristics of different types of experiments may be responsible for the inconsistency.

Statins have received increasing attention in clinical research in the field of various liver diseases including liver cirrhosis, hepatocellular carcinoma, fatty liver disease, viral hepatitis and other related liver diseases, in recent years. ${ }^{6}$ Studies have confirmed that statins are safe and effective for some patients with these liver diseases. ${ }^{41}$ A population-based study ${ }^{9}$ evaluated the effects of statins on reducing decompensation, mortality and hepatocellular carcinoma (HCC) in HBV, HCV and alcohol-related cirrhosis. This study demonstrated that statins reduced decompensation $(\mathrm{p}<0.0001)$, mortality $(p<0.0001)$ and the risk of HCC $(p=0.009)$ in patients with cirrhosis, and this correlation was dose-dependent. The risk of decompensation in patients with cirrhosis caused by chronic HBV (RR, 0.39 ; $95 \%$ CI, 0.25 to 0.62 ) or HCV infection (RR, $0.51 ; 95 \%$ CI, 0.29 to 0.93 ) was lower in patients taking statins. The effect of statins on reducing the risk of cirrhosis decompensation was statistically significant in alcohol cirrhotic patients (RR, $0.69 ; 95 \%$ CI, 0.45 to 1.07$)$. In general, the use of statins reduced the decompensation rate of $\mathrm{HBV}$, HCV and alcohol-related cirrhosis. Two recent studies ${ }^{42} 43$ demonstrated that statins were safe in patients with nonalcoholic fatty liver disease (NAFLD) and exhibited beneficial effects decreasing steatosis and fibrosis and preventing disease progression. Multiple previous studies demonstrated the benefit of statins on liver systems. A randomised trial of patients with cirrhosis and significant portal hypertension observed that the nitric oxide levels in hepatic venous blood, as a key vasodilator mediating the hepatic vascular resistance, ${ }^{44-46}$ were increased in the statin group compared with those in the control group. A decrease in portal hypertension was also observed in patients who received statins. ${ }^{47}$ The study by Marrone et $a t^{48}$ has also confirmed that the use of statins in cirrhotic animals can reduce liver fibrosis and prevent further deterioration of cirrhosis by inhibiting the activation of hepatic stellate cell. This may also be a potential mechanism for the efficacy of statins.

More research groups have begun to support the use of statin in some patients with chronic liver disease or cirrhosis based on these studies. ${ }^{49}{ }^{50}$ Some researchers believe that statins may also be able to be used as an adjuvant therapy in any chronic liver disease patients with indications for statin use to prevent decompensation or delay the progression of patients with decompensated cirrhosis. ${ }^{51}$ However, this information was derived from retrospective cohort studies, and prospective studies are needed to confirm these beneficial effects.
This meta-analysis evaluated the role of statins in patients with cirrhosis as a decline in portal pressure and risk of variceal haemorrhage. We performed a comprehensive literature search that met the well-defined inclusion criteria. Eight studies were included, primarily consisting of RCTs. These studies were high-quality studies as graded by the Cochrane tool for assessing risk of bias or the Newcastle-Ottawa scale. Several subgroup analyses were completed based on the characteristics of the studies to further ascertain the precision of results.

However, several limitations exist in our meta-analysis. In some of the results, we have a large heterogeneity, which may be due to the inconsistency of the inclusion and exclusion criteria we included in the study. In addition, patients with various aetiologies of cirrhosis were not researched separately because of insufficient information, which may explain the substantial heterogeneity. So we performed a subgroup analysis to try to eliminate this difference, significantly reducing heterogeneity in some subgroup analyses. Seven RCTs were included, but the number of patients enrolled was relatively fewer in the RCTs (471 patients). Although individual studies adjusted for various confounders (eg, age, sex, CTP score and model for end-stage liver disease (MELD) score), some confounding factors cannot be fully adjusted. These situations may have affected the precision and credibility of our estimates. In two studies, the 0-event counts in the control group may be due to the fact that the placebo used in the control group is not a drug such as NSBB that has been proven to have a reduced portal pressure, leading to a wide $95 \%$ CI. The quality assessment of the RCT suggests that the quality of the two studies is acceptable, so we have no good reason to exclude these studies. The number of patients included in some studies is insufficient, so continuity corrections is not used, which may increase the risk of bias. Non-alcoholic fatty liver disease, as a metabolic disease, may exhibit closer relevance with lipid-lowering drug statins. Unfortunately, no eligible NAFLD research was included.

In conclusion, our analyses based on RCTs and an observational study indicated a beneficial effect of statins on reducing portal hypertension and variceal haemorrhage. However, the assessment cannot serve as clinical guideline for the wide use of statins in cirrhosis with portal hypertension because of the limited quantity and quality of the included studies. Previous research reported the potential protective effects of statins against cirrhosis and HCC progression, and the potential benefits of statins may outweigh the theoretical risks. Notably, adverse events related to statins were rarely reported in studies. Large RCTs are required before statins are clinically used to treat patients with cirrhosis and complications.

Acknowledgements Thanks for the economic support from the National Natural Science Foundation of China.

Contributors SW and $\mathrm{CH}$ contributed equally to this study. SW planned the study. SW and $\mathrm{CH}$ screened the literature and collected data. SW, CH and XZ conducted the meta-analysis and wrote the manuscript. 
Funding This study was supported by the National Natural Science Foundation of China (grant number: 81660110), the "Gan-Po Talent 555" Project of Jiangxi Province, and the Nanchang University Graduate Innovation Special Fund Project (grant number: CX2018205).

Competing interests None declared.

Patient consent for publication Not required.

Provenance and peer review Not commissioned; externally peer reviewed.

Data sharing statement Data are available upon reasonable request.

Open access This is an open access article distributed in accordance with the Creative Commons Attribution Non Commercial (CC BY-NC 4.0) license, which permits others to distribute, remix, adapt, build upon this work non-commercially, and license their derivative works on different terms, provided the original work is properly cited, appropriate credit is given, any changes made indicated, and the use is non-commercial. See: http://creativecommons.org/licenses/by-nc/4.0/.

\section{REFERENCES}

1. Murray CJ, Atkinson C, Bhalla K, et al. The state of US health, 1990-2010: burden of diseases, injuries, and risk factors. JAMA 2013;310:591-608.

2. D'Amico G, Garcia-Tsao G, Pagliaro L. Natural history and prognostic indicators of survival in cirrhosis: a systematic review of 118 studies. $J$ Hepatol 2006;44:217-31.

3. D'Amico G, Luca A. Natural history. Clinical-haemodynamic correlations. Prediction of the risk of bleeding. Baillieres Clin Gastroenterol 1997;11:243-56.

4. Ripoll C, Groszmann R, Garcia-Tsao G, et al. Hepatic venous pressure gradient predicts clinical decompensation in patients with compensated cirrhosis. Gastroenterology 2007;133:481-8.

5. Sirtori CR. The pharmacology of statins. Pharmacol Res 2014;88:3-11.

6. Pose E, Trebicka J, Mookerjee RP, et al. Statins: Old drugs as new therapy for liver diseases? J Hepatol 2019;70.

7. Tsochatzis EA, Bosch J. Statins in cirrhosis-Ready for prime time. Hepatology 2017;66:697-9.

8. Moctezuma-Velázquez C, Abraldes JG, Montano-Loza AJ. The Use of Statins in Patients With Chronic Liver Disease and Cirrhosis. Curr Treat Options Gastroenterol 2018;16:226-40.

9. Chang FM, Wang YP, Lang $\mathrm{HC}$, et al. Statins decrease the risk of decompensation in hepatitis $B$ virus- and hepatitis $C$ virus-related cirrhosis: A population-based study. Hepatology 2017;66:896-907.

10. Abraldes JG, Villanueva C, Aracil C, et al. Addition of simvastatin to standard therapy for the prevention of variceal rebleeding does not reduce rebleeding but increases survival in patients with cirrhosis. Gastroenterology 2016;150:1160-70.

11. Mach F, Ray KK, Wiklund O, et al. Adverse effects of statin therapy: perception vs. the evidence - focus on glucose homeostasis, cognitive, renal and hepatic function, haemorrhagic stroke and cataract. Eur Heart J 2018;39:2526-39.

12. Shin JY, Azoulay L, Filion KB. Statin use in patients with hepatitis c-related cirrhosis: true benefit or immortal time bias? Gastroenterology 2016;151:373.

13. Chang $\mathrm{CH}$, Hsu YM, Chen YC, et al. Anti-inflammatory effects of hydrophilic and lipophilic statins with hyaluronic acid against LPSinduced inflammation in porcine articular chondrocytes. $J$ Orthop Res 2014;32:557-65.

14. Kolawole EM, McLeod JJ, Ndaw V, et al. Fluvastatin suppresses mast cell and basophil IgE Responses: genotype-dependent effects. $\mathrm{J}$ Immunol 2016;196:1461-70.

15. Feldt M, Bjarnadottir O, Kimbung S, et al. Statin-induced antiproliferative effects via cyclin D1 and p27 in a window-of-opportunity breast cancer trial. J Transl Med 2015;13:133.

16. Ramma W, Ahmed A. Therapeutic potential of statins and the induction of heme oxygenase-1 in preeclampsia. J Reprod Immunol 2014;101-102:153-60.

17. Oikonomou E, Siasos G, Zaromitidou M, et al. Atorvastatin treatment improves endothelial function through endothelial progenitor cells mobilization in ischemic heart failure patients. Atherosclerosis 2015;238:159-64.

18. Camargo LM, França CN, Izar MC, et al. Effects of simvastatin/ ezetimibe on microparticles, endothelial progenitor cells and platelet aggregation in subjects with coronary heart disease under antiplatelet therapy. Braz J Med Biol Res 2014;47:432-7.

19. Mehl A, Harthug S, Lydersen S, et al. Prior statin use and 90-day mortality in Gram-negative and Gram-positive bloodstream infection: a prospective observational study. Eur J Clin Microbiol Infect Dis 2015;34:609-17.

20. de Paula TP, Santos PC, Arifa R, et al. Treatment with atorvastatin provides additional benefits to imipenem in a model of gram-negative pneumonia induced by Klebsiella pneumoniae in Mice. Antimicrob Agents Chemother 2018;62.

21. Guyatt $G$, Oxman AD, Sultan $S$, et al. GRADE guidelines: 11. Making an overall rating of confidence in effect estimates for a single outcome and for all outcomes. J Clin Epidemiol 2013;66:151-7.

22. DerSimonian R, Laird N. Meta-analysis in clinical trials. Control Clin Trials 1986;7:177-88

23. Higgins JP, Thompson SG, Deeks JJ, et al. Measuring inconsistency in meta-analyses. BMJ 2003;327:557-60.

24. Mohanty A, Tate JP, Garcia-Tsao G. Statins are associated with a decreased risk of decompensation and death in veterans with Hepatitis C-Related Compensated Cirrhosis. Gastroenterology 2016;150:430-40.

25. Abraldes JG, Albillos A, Bañares R, et al. Simvastatin lowers portal pressure in patients with cirrhosis and portal hypertension: a randomized controlled trial. Gastroenterology 2009;136:1651-8.

26. Alvarado-Tapias E, Ardèvol A, Pavel O, et al. Hemodynamic effects of carvedilol plus simvastatin in cirrhosis with portal hypertension and no-response to $\beta$-blockers: A double-blind randomized trial. Hepatology 2016;64:74A.

27. Bishnu S, Ahammed SM, Sarkar A, et al. Effects of atorvastatin on portal hemodynamics and clinical outcomes in patients with cirrhosis with portal hypertension: a proof-of-concept study. Eur J Gastroenterol Hepatol 2018;30:54-9.

28. Flores PP, Rezende GF, Cassano U, et al. Effect of simvastatin in portal hypertension. Hepatology (Baltimore, Md) 2014;60:1191A.

29. Pollo-Flores P, Soldan M, Santos UC, et al. Three months of simvastatin therapy vs. placebo for severe portal hypertension in cirrhosis: A randomized controlled trial. Dig Liver Dis 2015;47:957-63.

30. Rajan V, Choudhary A, Jindal A, et al. Addition of simvastatin to carvedilol does not improve hemodynamic response in cirrhotics with varices without prior bleed: Preliminary results of an open label RCT. Hepatology 2016;64:1134A-5.

31. Bhardwaj SS, Chalasani N. Lipid-lowering agents that cause druginduced hepatotoxicity. Clin Liver Dis 2007;11:597-613.

32. Kubota T, Fujisaki K, Itoh Y, et al. Apoptotic injury in cultured human hepatocytes induced by HMG-CoA reductase inhibitors. Biochem Pharmacol 2004;67:2175-86.

33. Ballarè M, Campanini M, Airoldi G, et al. Hepatotoxicity of hydroxy-methyl-glutaryl-coenzyme A reductase inhibitors. Minerva Gastroenterol Dietol 1992;38:41-4.

34. Heuer T, Gerards H, Pauw M, et al. [Toxic liver damage caused by HMG-CoA reductase inhibitor]. Med Klin 2000;95:642-4.

35. Koornstra JJ, Ottervanger JP, Fehmers MC, et al. [Clinically manifest liver lesions during use of simvastatin]. Ned Tijdschr Geneeskd 1996;140:846-8.

36. Black DM, Bakker-Arkema RG, Nawrocki JW. An overview of the clinical safety profile of atorvastatin (lipitor), a new HMG-CoA reductase inhibitor. Arch Intern Med 1998;158:577-84.

37. Lebrec D, Poynard T, Bernuau J, et al. A randomised controlled study of propranolol for prevention of recurrent gastrointestinal bleeding in patients with cirrhosis. Drugs 1989;37(Suppl 2):30-4. discussion 47.

38. Hillon P, Lebrec D, Muńoz C, et al. Comparison of the effects of a cardioselective and a nonselective beta-blocker on portal hypertension in patients with cirrhosis. Hepatology 1982;2:528S-31.

39. Aramaki T, Sekiyama T, Katsuta Y, et al. Long-term haemodynamic effects of a 4-week regimen of nipradilol, a new beta-blocker with nitrovasodilating properties, in patients with portal hypertension due to cirrhosis. A comparative study with propranolol. $J$ Hepatol 1992;15(1-2):48-53.

40. Gatta A, Sacerdoti D, Merkel C, et al. Use of a nonselective betablocker, nadolol, in the treatment of portal hypertension in cirrhotics. Int J Clin Pharmacol Res 1985;5:413-8.

41. Pastori D, Polimeni L, Baratta F, et al. The efficacy and safety of statins for the treatment of non-alcoholic fatty liver disease. Dig Liver Dis 2015;47:4-11.

42. Dongiovanni P, Petta S, Mannisto V, et al. Statin use and non-alcoholic steatohepatitis in at risk individuals. J Hepatol 2015;63:705-12.

43. Nascimbeni F, Aron-Wisnewsky J, Pais R, et al. Statins, antidiabetic medications and liver histology in patients with diabetes with non-alcoholic fatty liver disease. BMJ Open Gastroenterol 2016;3:e000075.

44. Gupta TK, Toruner M, Chung MK, et al. Endothelial dysfunction and decreased production of nitric oxide in the intrahepatic microcirculation of cirrhotic rats. Hepatology 1998;28:926-31. 
45. Rockey DC, Chung JJ. Reduced nitric oxide production by endothelial cells in cirrhotic rat liver: endothelial dysfunction in portal hypertension. Gastroenterology 1998;114:344-51.

46. Shah V, Toruner M, Haddad F, et al. Impaired endothelial nitric oxide synthase activity associated with enhanced caveolin binding in experimental cirrhosis in the rat. Gastroenterology 1999;117:1222-8.

47. Zafra C, Abraldes JG, Turnes J, et al. Simvastatin enhances hepatic nitric oxide production and decreases the hepatic vascular tone in patients with cirrhosis. Gastroenterology 2004:126:749-55.
48. Marrone G, Maeso-Díaz R, García-Cardena G, et al. KLF2 exerts antifibrotic and vasoprotective effects in cirrhotic rat livers: behind the molecular mechanisms of statins. Gut 2015;64:1434-43.

49. Lin CY. Statins and risk of decompensation in hepatitis B virusrelated and hepatitis $C$ virus-related cirrhosis: Methodological issues. Hepatology 2018;67:1174

50. Kim G, Jang SY, Nam CM, et al. Statin use and the risk of hepatocellular carcinoma in patients at high risk: A nationwide nested case-control study. J Hepatol 2018;68:476-84.

51. Magan-Fernandez A, Rizzo M, Montalto G, et al. Statins in liver disease: not only prevention of cardiovascular events. Expert Rev Gastroenterol Hepatol 2018;12:743-4. 